\title{
Gestational Diabetes Mellitus: A Review Article
}

\author{
P.Rajalakshmy
}

\section{Abstract:}

Gestational diabetes mellitus (GDM) is defined as glucose intolerance that begins or is first detected during pregnancy. About $7 \%$ of all pregnancy is $>200,000$ cases per year are detected as GDM. GDM will be diagnosed through oral glucose tolerance test (OGTT). It can be identified in the first trimester. Since this GDM will increase the complication among the mother and the infant. Since it cannot be curable but the complication can be reduced with the special care, drug and the dietary management.

Key words: Gestational Diabetes Mellitus (GDM); Glycemic Index; Glycemic Load

\section{INTRODUCTION}

India rank number one position in type II diabetes Mellitus. Diabetes Mellitus can be coined as hyperglycemia that means increased blood glucose level seen in the period of pregnancy is termed as Gestational Diabetes Mellitus ${ }^{1}$

Where GDM is defined as imbalanced glucose metabolism in the period of pregnancy detected during first trimester, based on the recent study it shows that GDM affects $>200,00$ cases per year. ${ }^{2}$ GDM Occurs in 2- 9\% of all pregnancy associated with maternal and perinatal complication $^{3-4}$ the incidence of GDM is being raised mainly based on obesity level in the population ${ }^{5}$.

\section{AIMS AND OBJECTIVE}

The aim of this review article is to elaborate about the treatments to reduce the complication of theGDM.

\section{Complications}

Since many study shows that GDM affected women and the infant born with
GDM women, were facing many complication even in their future. The GDM pregnancy and birth itself will have an increased risk of complications for both mother andbaby. ${ }^{6}$

\section{Complication in Mothers with GDM}

The GDM women have an increased risk of developing type II diabetes mellitus after pregnancy

\section{Complication in Infants with GDM mothers}

The major complication faced by the infant with the GDM mothers are macrosomia, Neonatal hypoglycemia, perinatal mortality ,congenital malformation, hyper-bilirubinemia, polycythemia , hypocalcemia and respiratory distress syndrome excess in fetal growth can leads to increased fetal morbidity at delivery. Neonatal hypoglycemia seen in newborn within few hours after delivery is because of hyperglycemia that causes hyperinsulinemia in infants. Several studies have concluded that the rate ofperinatal 
mortality was increased in women with GDM. Ketonemia in mothers with GDM associate with lower IQ levels and impaired psychomotor development in their children. Untreated GDM is noted to increase the risk of still birth by approximately fourfold. ${ }^{6}$

\section{Treatment for GDM}

Recent studies have shown that, Increased Antepartum Monitoring, Medical Nutrition Therapy and Insulin Therapy reduce the risk of GDM. The US diabetes prevention programme (DPP) stated that lifestyle changes works women pos- GDM reduce the risk of type II diabetes mellitus by $50 \%$. Lifestyle modification is warranted to prevent diabetes mellitus, the guidelines for physical activity are relatively consistent but it is the area of nutrition where the difference is more evident. Since much study prove that showing special attention in care giving, Drugs and Nutritional Support will reduce the complication of GDM, a few have discussed.

\section{Comparative study of Intensive and Routine - Care:}

Care is an important to reduce the complication of GDM. While comparing the complication of the intensive and routine hospital care.

No perinatal deaths occur in intensive care group, were 0.98 percent perinatal death in routine- care group. No infants in the intensive care group had bone fracture or nerve palsy. Whereas 0.19 percent infant had both fractured humerus and radial-nerve palsy, one infant had erb's palsy alone in the routine- care group. Infants born in intensive care group has lower mean birth weight than the infants born to routine- care group women. As a result of this study proved that intensive care among GDM women will reduce the risk and complication for the infants and mother. ${ }^{6}$

\section{Impact of Glycemic Index(GI) and Glycemic Load(GL) amongGDM}

GI need to be considered in the relationship between quality of $\mathrm{CHO}$ alter the postprandial glycemia.GDM diet often focuses on the relationship between quality of $\mathrm{CHO}$ and post prandial glycemia, where the higher nutritional requirement of pregnancy may be underemphasized GI is an important factor for GDM women.

According to the study women who consuming lower GL diet had improved intake of riboflavin, potassium, calcium and vitamin $\mathrm{A}, \mathrm{D}, \& \mathrm{E}$ where as those who consume more grains and cereals higher in Thus lower dietary GI product had poor diet quality and lower intake of and GL associated with superior dietary quality ${ }^{5-7}$

\section{CONCLUSION}

GDM is fairly common problem in the present era. Timely diagnosed and treatment reduces the risk of severe complications, based on the recent study it proved that intensive care and less intake of GI and GL rich food will reduce the risk and complication among mother and the infant even in the future.

\section{REFERENCE}

1.S.Wild, G.Roglic, A.Green, R.Sicree and H.king (2004), “ Global prevalence of diabetes : estimates for the year 2000 and projection for 2030," Diabetes Care vol.27, pp.2047-1053.

doi:10.2337/diacare.27.10.2568.

2. American Diabetes Association: Gestational diabetes mellitus (Position Statement). Diabetes Care 27 (Suppl. 1):S88-S90,2004.

3. L.Hoffman, C.Nolan, J. D.Wilson, J. Oats, and D.Simmons, "Gestational diabetes mellitus - management guidelines", The 
Australasian Diabetes in Pregnancy Society. Med J Aust 1998;vol.169, pp.937.PMID:9700346

4.Clinical management guidelines for obstetrician-gynecologists. ACOG practice bulletin no. 30. Washington, D.C.: American College of Obstetricians and Gynecologists, 2001

5. K.J Hunt and K.L Schuller, "The increasing prevalence of diabetes in pregnancy", Obstet. Gynecol. Clin. North. Am. 2007, vol.34, pp.173-199. doi: 10.1016/j.ogc.2007.03.002.
6. B.R Shah, R. Retnakaran, G.L. Booth,"Increased risk of cardiovascular disease in young women following gestational diabetes mellitus," Diabetes Care 2008,vol.31, pp.1668-1669.

doi:10.2337/dc08-0706

7. G.D .Cianni, R.Miccoli, L.Volpe, C.Lencioni, S.Del Prato, "Intermediate metabolism in nomal pregnancy and in gestational diabetes," Diabetes Metab Res Rev vol.19, pp.259-270, 2003. doi:10.1002/dmrr.390.

\section{Miscarriage: recurrent miscarriage causes}

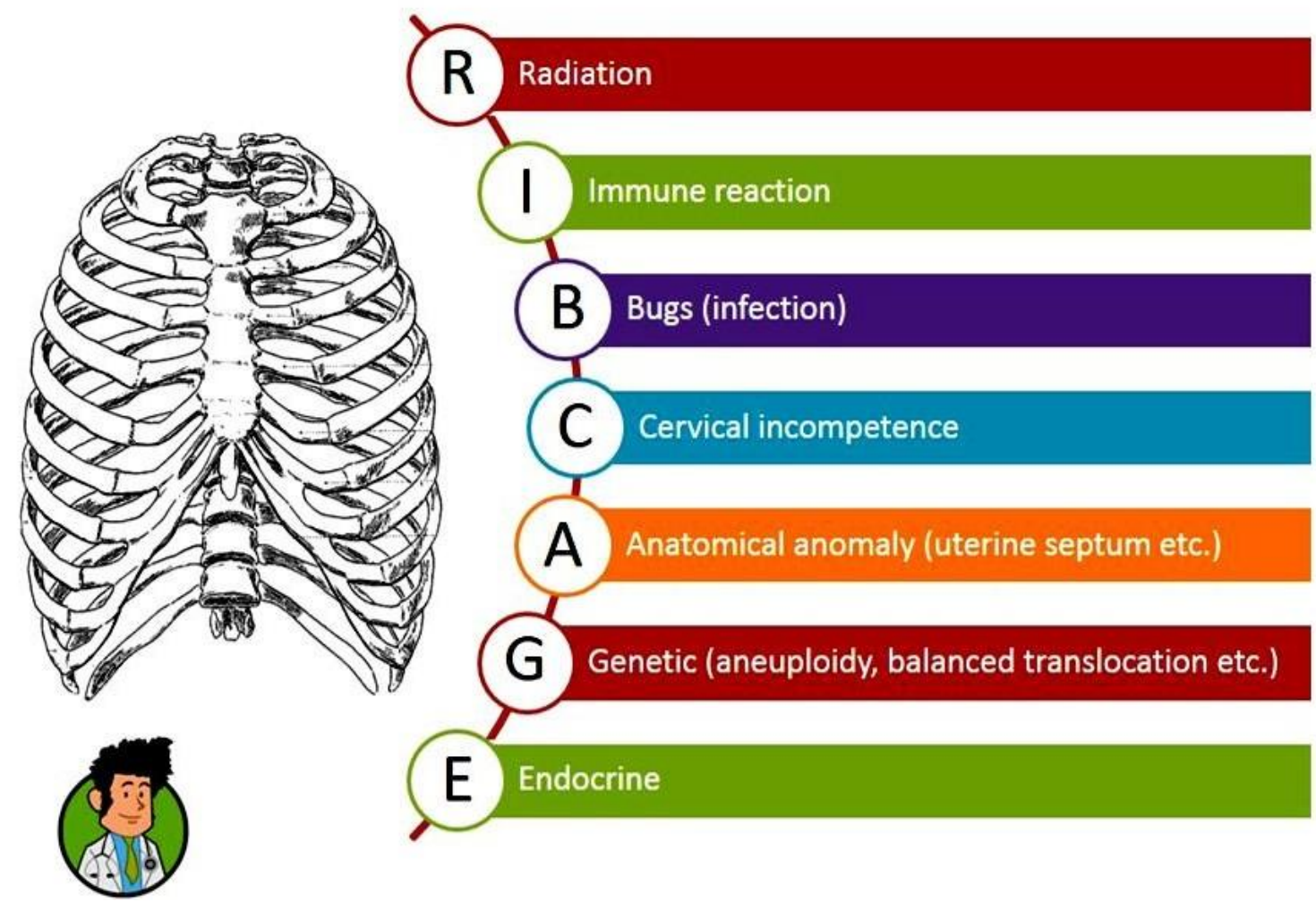

